\title{
Modelling the Blocking Behavior of Multicast Clos Networks
}

\author{
Achille Pattavina and Gino L. Tesei \\ Politecnico di Milano \\ Dept. of Electronics and Information \\ P.zza L. Da Vinci 32, 20133 Milano, ITALY \\ Email: pattavina@elet.polimi.it
}

\begin{abstract}
This paper considers three-stage switching networks able to support multicast traffic, i.e. connections in which one inlet is connected to more than one output at the same time. The nonblocking conditions for this network are studied under the assumption of absence of any optimized routing of the connections inside the structure (the so-called strict-sense nonblocking networks). An analytical model is developed here that provides not only the nonblocking conditions of three-stage multicast networks, but also the evaluation of the blocking probability when such conditions are not satisfied. Unlike previous wellknown approaches, our model takes into account the correlation between occupancy events in links belonging to different interstage patterns. The results being found also provide a more stringent condition of network nonblocking for multicast traffic which disproves some of the claimed results recently published in the technical literature.
\end{abstract}

\section{INTRODUCTION}

The first breakthrough on how to build minimum-cost multistage connecting networks starting from small crossbars has been known since 1953 and is due to Charles Clos [1]. These three-stage networks, nowadays known as Clos networks, have been widely used for data communications and parallel computing systems under the assumption that the service supported is always unicast, that is each connection is established between an idle inlet and only one idle outlet of the network. Interest in this kind of networks has been raising in the last two decades due to the growing needs for supporting multicast communication services, which implies that a switching node must be able to set-up connections from an idle inlet to more than one idle outlet. In this paper we give the necessary and sufficient conditions for a three-stage switching network to be strictly nonblocking when each inlet can address a number of outlets ranging from a minimum $f_{1}$ up to a maximum $f_{2}$. Our model evaluates also the blocking probability when the network configuration parameters determine a blocking structure. We assume that the multicast connection in set-up as a single operation.

It is worth reminding that the nonblocking Clos network does not set any constraint on how to select the new connection out of the multiple paths available through the three-stage network. Such class of networks, called strict-sense nonblocking networks (SSNB), must be distinguished from other two types of nonblocking networks, i.e. the wide-sense nonblocking networks (WSNB) and the rearrangeable nonblocking networks
(RNB). In both cases a less complex nonblocking network is built compared to the SSNB one by allowing, at connection set-up time, either an optimized path selection or a possible rearrangement of already set-up connections. In this paper we will only consider the case of SSNB networks.

Our approach extends to the multicast case the analytical model developed in [2] for evaluating the blocking probability of unicast three-stage switching networks. This model, unlike traditional analytical models such as Lee's model [3], is exact in that it takes into account the dependence between occupancy events of links in different interstage patterns. Hence it is consistent with the deterministic nonblocking conditions such as the Clos condition [1]. The nonblocking conditions derived here confirm and extend the results given in [4], in which the authors consider a less general case of fanout variability. Our results on nonblocking conditions disprove also the necessity of previously published results [5] claiming to be both necessary and sufficient (for a discussion on this point, see [6]).

In the following, Section 2 prepares the mathematical background needed in the paper. The model for evaluating the blocking probability in a three-stage network is developed in Section 3. Sufficiency and necessity of nonblocking conditions are proven in Sections 4 and 5. Finally some numerical results are discussed in Section 6.

\section{Mathematical Preliminaries}

\section{A. Multicast 3-stage switching networks}

Definition 1: Let us consider an $N \times N$ multicast switching network, i.e. in which each inlet can address a nonnegative number $f_{i}$ of outlets, where $f_{1} \leq f_{i} \leq f_{2}$. Obviously, we assume that all matrices are multicast, i.e. in which each inlet can be connected to more than one outlet at the same time. The network structure under consideration is shown in Fig. 1: it has $N$ inlets, $N$ outlets and it is composed of three stages of crossbar matrices with size $n \times m$ at stage $1, r \times r$ at stage $2, m \times n$ at stage 3 , where $r=N / n$. We denote this class of networks by $\operatorname{M-CLOS}\left(N, n, m ; f_{1}, f_{2}\right)$.

Definition 2: In an M-CLOS $\left(N, n, m ; f_{1}, f_{2}\right)$
the set of inlets and outlets are denoted by
$I=\left\{i_{k, i} \mid k \in\{1, \ldots, r\} \wedge i \in\{1, \ldots, n\}\right\}$ and $O$ =
$\left\{o_{h, j} \mid h \in\{1, \ldots, r\} \wedge j \in\{1, \ldots, n\}\right\}$, respectively, the
set of inlets of the $k$-th matrix at first stage is denoted by




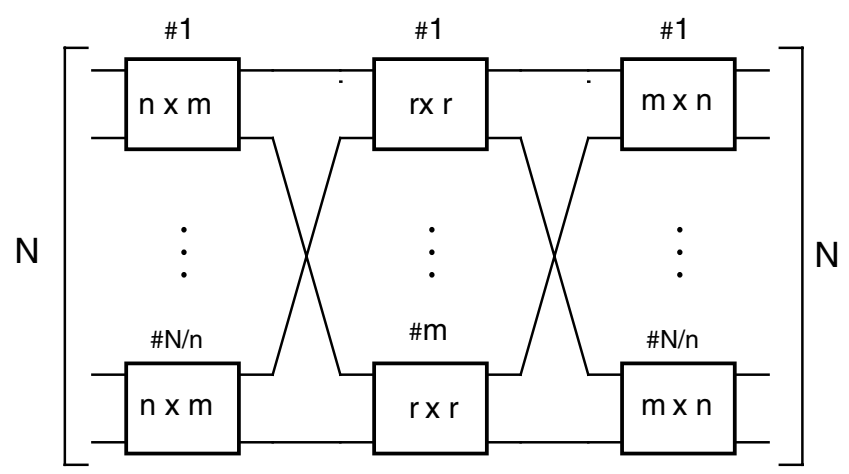

Fig. 1. Configuration of 3-stage switching network

$I_{k}=\left\{i_{k, i} \mid i \in\{1, \ldots, n\}\right\}$, where $k \in\{1, \ldots, r\}$, the set of outlets of the $h$-th matrix at third stage is denoted by $O_{h}=\left\{o_{h, j} \mid j \in\{1, \ldots, n\}\right\}$, where $h \in\{1, \ldots, r\}$.

Definition 3: Given an inlet $i_{k, i}$, let $C_{1, k, i}$ be the set of inlets defined as

$$
C_{1, k, i}=I_{k}-\left\{i_{k, i}\right\}
$$

and, given an outlet $o_{h, j}$, let $C_{2, h, j}$ be the set of outlets defined as

$$
C_{2, h, j}=O_{k}-\left\{o_{h, j}\right\} .
$$

Definition 4: In an M-CLOS ( $\left.N, n, m ; f_{1}, f_{2}\right)$ the fanout of the inlet $i_{k, i}$ is denoted by $f_{k, i}$, where

$$
\left\{\begin{array}{cl}
f_{1} \leq f_{k, i} \leq f_{2} & \text { if } i_{k, i} \text { is busy } \\
f_{k, i}=0 & \text { otherwise. }
\end{array}\right.
$$

Definition 5: According to the above definitions, in an $\operatorname{M-CLOS}\left(N, n, m ; f_{1}, f_{2}\right)$ the multicast connection request from the inlet $i_{k, i}$, provided that $f_{k, i} \in\left\{f_{1}, \ldots, f_{2}\right\}$, to the set of outlets $O_{k, i} \subseteq O$, is denoted by $C\left(i_{k, i} ; O_{k, i}\right)$. Notice that $\left|O_{k, i}\right|=f_{k, i}$. Moreover, we denote by $c\left(i_{k, i}, o_{h, j}\right)$, where $o_{h, j} \in O_{k, i}$, one of the point-to-point connection requests belonging to $C\left(i_{k, i} ; O_{k, i}\right)$.

Lemma 1: For each M-CLOS $\left(N, n, m ; f_{1}, f_{2}\right)$ the maximum number of addressed outlets by $k$ free inlets $(k \leq N)$, provided that there are already $h$ free outlets $(h \leq N)$, is given by

$$
A_{\text {max }}\left(k, h ; f_{1}, f_{2}\right)=\min \left\{k f_{2}, h,\left\lfloor\frac{h}{f_{1}}\right\rfloor f_{2}\right\} .
$$

Proof: The proof of this lemma is given in [6].

\section{B. Preliminaries of discrete mathematics}

Definition 6: The quantities $x^{\underline{k}}$ and $x^{\bar{k}}$ (read " $x$ to the $k$ falling" and " $x$ to the $k$ rising"), when $k$ is a positive integer, are defined as follows

$$
x^{\underline{k}}=\prod_{0 \leq j \leq k-1}(x-j)
$$

and

$$
x^{\bar{k}}=\prod_{0 \leq j \leq k-1}(x+j) .
$$

Definition 7: For each $r \in \mathbb{R}$ and $k$ integer, we define

$$
\left(\begin{array}{l}
r \\
k
\end{array}\right)=\left\{\begin{array}{cc}
\frac{r \underline{\underline{k}}}{k !} & k \geq 0 \\
0 & k<0
\end{array} .\right.
$$

Lemma 2: For each $r, n \in \mathbb{N}$, we have

$$
\sum_{0 \leq k \leq \min \{n, r\}}\left(\begin{array}{l}
r \\
k
\end{array}\right)\left(\begin{array}{c}
s \\
n-k
\end{array}\right)=\left(\begin{array}{c}
r+s \\
n
\end{array}\right)
$$

Proof: According to Vandermonde's convolution [7, p.29], i.e.

$$
\sum_{k}\left(\begin{array}{l}
r \\
k
\end{array}\right)\left(\begin{array}{c}
s \\
n-k
\end{array}\right)=\left(\begin{array}{c}
r+s \\
n
\end{array}\right), n \text { integer, }
$$

as $r \in \mathbb{N}$, we argue from definition 7 that

(i) $\left(\begin{array}{l}r \\ k\end{array}\right)=0$ for $r<k \Longrightarrow k \leq r$,

(ii) $\left(\begin{array}{l}r \\ k\end{array}\right)=0$ for $k<0 \Longrightarrow 0 \leq k$,

(iii) $\left(\begin{array}{c}s \\ n-k\end{array}\right)=0$ for $n-k<0 \Longrightarrow k \leq n$.

Consequently, equation (1) can be rewritten as

$$
\sum_{0 \leq k \leq \min \{n, r\}}\left(\begin{array}{l}
r \\
k
\end{array}\right)\left(\begin{array}{c}
s \\
n-k
\end{array}\right)=\left(\begin{array}{c}
r+s \\
n
\end{array}\right) .
$$

\section{MOdEL FOR MULTICAST CONNECTIONS}

Let us consider an $\operatorname{M-CLOS}\left(N, n, m ; f_{1}, f_{2}\right)$, provided that the set of outlets referenced by $i_{k, i}$ includes $o_{h, j}$, i.e. we consider the multicast connection request $C\left(i_{k, i} ; O_{k, i}\right)$ where $c\left(i_{k, i}, o_{h, j}\right)\left(o_{h, j} \in O_{k, i}\right)$ is one of the point-to-point connection requests in which $C\left(i_{k, i} ; O_{k, i}\right)$ can be ideally decomposed. Hence,

- $N_{1}$ denotes the event in which there are $n_{1}$ busy inputmiddle interstage links allocated to the connections established by the set of inlets $C_{1, k, i}$;

- $N_{2}$ denotes the event in which there are $n_{2}$ busy middleoutput interstage links allocated to the connections directed to the set of of outlets $C_{2, h, j}$;

- the pairs of input-middle interstage and middle-output interstage links sharing the same middle stage switch are said to be overlapped.

Moreover,

1) the events that individual links in the same interstage pattern are busy are assumed to be independent;

2) traffic load is uniformly distributed and uniformly addressed; let $p \in[0,1]$ be the probability that an interstage link is busy, and $q=1-p$ the probability that an interstage link is not busy; given the expansion ratio $m / n$ at the first stage, then $p=a \cdot n / m$, if $a$ is the probability that an external link is busy; 
3) the number of busy input-middle (and middle-output) interstage links follows the binomial distribution.

Lemma 3: Given the events $N_{1}$ and $N_{2}$ under the hypotheses $1,2,3$, the probability that $k$ pairs of interstage links are overlapped in an M-CLOS $\left(N, n, m ; f_{1}, f_{2}\right)$ network is given by

$$
\left.\begin{array}{c}
\operatorname{Pr}\left\{k \text { pairs of links overlapped } \mid N_{1}, N_{2}\right\}= \\
\left(\begin{array}{c}
n_{1} \\
k
\end{array}\right)\left(\begin{array}{c}
m-n_{1} \\
n_{2}-k
\end{array}\right) \\
n_{2}
\end{array}\right)=\frac{\left(\begin{array}{c}
n_{2} \\
k
\end{array}\right)\left(\begin{array}{c}
m-n_{2} \\
n_{1}-k
\end{array}\right)}{\left(\begin{array}{c}
m \\
n_{1}
\end{array}\right)} .
$$

Proof: The proof is given in [2] in case of one-toone connections. The fact that connections are one-to-many implies no differences.

Thus, given the events $N_{1}$ and $N_{2}$, provided that there are $k$ overlapped links, the connection request $c\left(i_{k, i}, o_{h, j}\right)$ is not blocked if, and only if, $n_{1}+n_{2}-k<m$. Notice that the number of overlapped links must be lower both than the number of the busy input-middle interstage links $\left(n_{1}\right)$ and than the number of the busy middle-output interstage links $\left(n_{2}\right)$, i.e. $k \leq \min \left\{n_{1}, n_{2}\right\}$. Thus the probability that the connection request $c\left(i_{k, i}, o_{h, j}\right)$ is satisfied, given the events $N_{1}, N_{2}$, is

$$
\begin{aligned}
& \operatorname{Pr}\left\{\text { connection not blocked } \mid N_{1}, N_{2}\right\}= \\
& =\frac{1}{\left(\begin{array}{c}
m \\
n_{2}
\end{array}\right)} \sum_{\delta_{1} \leq k \leq \delta_{2}}\left(\begin{array}{c}
n_{1} \\
k
\end{array}\right)\left(\begin{array}{c}
m-n_{1} \\
n_{2}-k
\end{array}\right),
\end{aligned}
$$

where $\delta_{1}=\max \left\{0, n_{1}+n_{2}-m+1\right\} \quad$ and $\delta_{2}=\min \left\{n_{1}, n_{2}\right\}$. Moreover, by the hypothesis 1 ,

$$
\operatorname{Pr}\left\{N_{1}, N_{2}\right\}=\operatorname{Pr}\left\{N_{1}\right\} \cdot \operatorname{Pr}\left\{N_{2}\right\} .
$$

In case of one-to-one connections, as shown in [2], there are at most $n-1$ busy input-middle interstage links from $C_{1, k, i}$, since each inlet has an unitary fanout by definition. On the contrary, in case of one-to-many connections the following lemma holds.

Lemma 4: Given the multicast connection request $C\left(i_{k, i} ; O_{k, i}\right)$, in an $\operatorname{M-CLOS}\left(N, n, m ; f_{1}, f_{2}\right)$ network there are at most $\overline{m_{1, k, i}}$ busy input-middle interstage links from $C_{1, k, i}$, where

$$
\overline{m_{1, k, i}}=\min \left\{(n-1) f_{2}, N-f_{1},\left(\left\lfloor\frac{N}{f_{1}}\right\rfloor-1\right) f_{2}\right\} .
$$

Proof: It is obvious that

$$
\overline{m_{1, k, i}} \leq(n-1) f_{2},
$$

since $\left|C_{1, k, i}\right|=n-1$ and the maximum fanout is $f_{2}$ for each inlet. Nevertheless this is only an upper bound. In fact, the set of inlets $C_{1, k, i}$ can reference the maximum number of free outlets if "the conspired inlet" $i_{k, i}$ has the minimum possible fanout, i.e. $f_{1}$. Hence, the maximum number of outlets that
$C_{1, k, i}$ can reference is $N-f_{1}$ and $\overline{m_{1, k, i}}$ coincides with the maximum number of addressed outlets by $n-1$ free inlets, provided that there are already $N-f_{1}$ free outlets, i.e.

$$
\overline{m_{1, k, i}}=A_{\max }\left(n-1, N-f_{1} ; f_{1}, f_{2}\right),
$$

which, according to lemma 1 , is given by

$$
\overline{m_{1, k, i}}=\min \left\{(n-1) f_{2}, N-f_{1},\left(\left\lfloor\frac{N}{f_{1}}\right\rfloor-1\right) f_{2}\right\},
$$

Notice that $\overline{m_{1, k, i}}$, that was here defined for $i_{k, i}$, is the same for each inlet and for each matrix at first stage; hence, let $\overline{m_{1, k, i}}=\bar{m}$. So, by hypotheses 2 and 3 ,

$$
\operatorname{Pr}\left\{N_{1}\right\}=\frac{\left(\begin{array}{c}
m \\
n_{1}
\end{array}\right) p^{n_{1}} q^{m-n_{1}}}{\sum_{0 \leq j \leq \bar{m}}\left(\begin{array}{c}
m \\
j
\end{array}\right) p^{j} q^{m-j}} .
$$

On the contrary, as in unicast switching networks, the largest number of busy links at second interstage, in order to realize the connection requests addressed to $C_{2, h, j}$, is still $n-1$; then it follows that

$$
\operatorname{Pr}\left\{N_{2}\right\}=\frac{\left(\begin{array}{c}
m \\
n_{2}
\end{array}\right) p^{n_{2}} q^{m-n_{2}}}{\sum_{0 \leq j \leq n-1}\left(\begin{array}{c}
m \\
j
\end{array}\right) p^{j} q^{m-j}}
$$

The previous discussion is performed given events $N_{1}$ and $N_{2}$, i.e. under the assumption that there are $n_{1}$ busy inputmiddle interstage links allocated to the connections established by the set of inlets $C_{1, k, i}$ and $n_{2}$ busy middle-output interstage links allocated to the connections directed to the set of of outlets $C_{2, h, j}$. Now let us focus on the problem concerning the maximum number of busy input-middle and middle-output interstage links without considering possible overlapped links. The following lemma holds.

Lemma 5: Given the multicast connection request $C\left(i_{k, i} ; O_{k, i}\right)$ and according to the above definitions,

$$
\begin{gathered}
n_{1}+n_{2} \leq \min \left\{(n-1) f_{2}+n-1,\right. \\
\left.N-f_{1},\left(\left\lfloor\frac{N}{f_{1}}\right\rfloor-1\right) f_{2}\right\}=\bar{\Omega} .
\end{gathered}
$$

Proof: From $0 \leq n_{1} \leq \bar{m}$ and $0 \leq n_{2} \leq n-1$, we obtain $n_{1}+n_{2} \leq(n-1) f_{2}+n-1$, if $\overline{m_{1, k, i}}=(n-1) f_{2}$. On the contrary, if $\bar{m}=\min \left\{N-f_{1},\left(\left\lfloor\frac{N}{f_{1}}\right\rfloor-1\right) f_{2}\right\}$ and, hence, $(n-1) f_{2}+n-1 \geq(n-1) f_{2} \geq$ $\min \left\{N-f_{1},\left(\left\lfloor\frac{N}{f_{1}}\right\rfloor-1\right) f_{2}\right\}$, then there are no more free outlets in order to set up the connections corresponding to the $n_{2}$ busy middle-output interstage links allocated to the connections directed to the set of outlets $C_{2, h, j}$; i.e. $n_{2}=0$, since $i_{k, i}$ needs at least $f_{1}$ free outlets by definitions. Notice that $n_{1}+n_{2}$ is maximal if $f_{k, i}$ is minimal, i.e. $f_{k, i}=f_{1}$. 
In conclusion, the nonblocking probability for each connection request becomes

$$
P_{N B}=\frac{\sum_{\substack{0 \leq n_{1} \leq m \\
0 \leq n_{2} \leq n-1}} \sum_{\delta_{1} \leq k \leq \delta_{2}}\left(\begin{array}{c}
m \\
n_{1}
\end{array}\right)\left(\begin{array}{c}
m-n_{1} \\
n_{2}-k
\end{array}\right)\left(\begin{array}{c}
n_{1} \\
k
\end{array}\right) p^{n_{1}+n_{2} q^{2 m-n_{1}-n_{2}}}}{\left[\sum_{0 \leq j \leq n-1}\left(\begin{array}{c}
m \\
j
\end{array}\right) p^{j} q^{m-j}\right]\left[\sum_{0 \leq i \leq m}\left(\begin{array}{c}
m \\
i
\end{array}\right) p^{i} q^{m-i}\right],}
$$

where $\delta_{1}=\max \left\{0, n_{1}+n_{2}-m+1\right\} \quad$ and $\delta_{2}=\min \left\{n_{1}, n_{2}\right\}$, while the blocking probability is

$$
P_{B}=1-P_{N B}
$$

This concludes the multicast extension of the analytical model by Yang and Kessler. The next section focuses on the application of such extended model under the deterministic nonblocking condition derived in [6], confirming its sufficiency.

\section{PROOF OF SUFFICIENCY}

It is proved in [6] that a non-squared $N_{1} \times N_{2}$ Clos network with $n_{1}$ inlets per first-stage matrix, $n_{2}$ outlets per third-stage matrix is SSNB, if and only if

$$
\begin{gathered}
m \geq \min \left\{\left(n_{1}-1\right) f_{2}+n_{2},\left(N_{1}-1\right) f_{2}+1,\right. \\
\left.N_{2}-f_{1}+1,\left(\left\lfloor\frac{N_{2}}{f_{1}}\right\rfloor-1\right) f_{2}+1\right\}
\end{gathered}
$$

that, in case of an M-CLOS $\left(N, n, m ; f_{1}, f_{2}\right)$, becomes

$$
\begin{gathered}
m \geq \min \left\{(n-1) f_{2}+n, N-f_{1}+1,\right. \\
\left.\left(\left\lfloor\frac{N}{f_{1}}\right\rfloor-1\right) f_{2}+1\right\} .
\end{gathered}
$$

The following lemma is crucial.

Lemma 6: Given $C\left(i_{k, i} ; O_{k, i}\right)$ and $\left|O_{k, i}\right|=f_{k, i}=f_{1}$, then $\delta_{1}=\max \left\{0, n_{1}+n_{2}-m+1\right\}=0$ if

$$
\begin{gathered}
m \geq \min \left\{(n-1) f_{2}+n, N-f_{1}+1,\right. \\
\left.\left(\left\lfloor\frac{N}{f_{1}}\right\rfloor-1\right) f_{2}+1\right\} .
\end{gathered}
$$

Proof: The proof of this lemma is given in Appendix.

In case of one-to-one connections the analytical model in [2] provides a null blocking probability, if $m \geq 2 n-1$ (Clos theorem). Thus, in case of one-to-many connections the multicast extended model should provide a null blocking probability if $m \geq \min \left\{(n-1) f_{2}+n, N-f_{1}+1,\left(\left\lfloor\frac{N}{f_{1}}\right\rfloor-1\right) f_{2}+1\right\}$. In fact, the following theorem holds.
Theorem 1: $P_{B}=0$ if

$$
\begin{gathered}
m \geq \min \left\{(n-1) f_{2}+n, N-f_{1}+1,\right. \\
\left.\left(\left\lfloor\frac{N}{f_{1}}\right\rfloor-1\right) f_{2}+1\right\},
\end{gathered}
$$

$$
\text { Proof: By lemma }
$$
$\delta_{1}=\max \left\{0, n_{1}+n_{2}-m+1\right\}=0$, hence

$$
\frac{1}{\left(\begin{array}{c}
m \\
n_{2}
\end{array}\right)} \sum_{0 \leq k \leq \min \left\{n_{1}, n_{2}\right\}}\left(\begin{array}{c}
n_{1} \\
k
\end{array}\right)\left(\begin{array}{c}
m-n_{1} \\
n_{2}-k
\end{array}\right)=1
$$

by lemma 2 . Thus, the relationship (6) becomes

$$
P_{N B}=\frac{\sum_{\substack{0 \leq n_{1} \leq \bar{m} \\
0 \leq n_{2} \leq n-1}}\left(\begin{array}{c}
m \\
n_{1}
\end{array}\right)\left(\begin{array}{c}
m \\
n_{2}
\end{array}\right) p^{n_{1}+n_{2}} q^{2 m-n_{1}-n_{2}}}{\left[\sum_{0 \leq j \leq n-1}\left(\begin{array}{c}
m \\
j
\end{array}\right) p^{j} q^{m-j}\right]\left[\sum_{0 \leq i \leq \bar{m}}\left(\begin{array}{c}
m \\
i
\end{array}\right) p^{i} q^{m-i}\right]}=1,
$$

which implies

$$
P_{B}=1-P_{N B}=0
$$

This concludes the check that the condition derived in [6] is at least sufficient in order to build SSNB 3-stage networks. On the contrary, the next section will check that such condition is also necessary; i.e. assuming

$$
\begin{aligned}
m<\min & \left\{(n-1) f_{2}+n, N-f_{1}+1,\right. \\
& \left.\left(\left\lfloor\frac{N}{f_{1}}\right\rfloor-1\right) f_{2}+1\right\}
\end{aligned}
$$

there is at least a network belonging to the class of $\operatorname{M-CLOS}\left(N, n, m ; f_{1}, f_{2}\right)$, so that the extended analytical model here derived provides a non-null blocking probability.

\section{PROOF OF NECESSITY}

Let us consider an $\operatorname{M-CLOS}\left(N, n, m ; f_{1}, f_{2}\right)$, so that

$$
\begin{gathered}
m<\min \left\{(n-1) f_{2}+n, N-f_{1}+1,\right. \\
\left.\left(\left\lfloor\frac{N}{f_{1}}\right\rfloor-1\right) f_{2}+1\right\} .
\end{gathered}
$$

Let

1) $f_{1}=1$

2) $n, N, f_{2}$ such that $(n-1) f_{2}+n \leq N-f_{1}+1$ and $\quad(n-1) f_{2}+n \leq\left(\left\lfloor\frac{N}{f_{1}}\right\rfloor-1\right) f_{2}+1 ; \quad$ for instance, if $\quad N=1000, n=10, f_{2}=5 \quad$ then $(n-1) f_{2}+n=55<999=N-f_{1} \quad$ and $(n-1) f_{2}+n=55<4996=\left(\left\lfloor\frac{N}{f_{1}}\right\rfloor-1\right) f_{2}+1$.

According to the above assumptions, it follows that

$$
\begin{aligned}
\bar{m} & =\min \left\{(n-1) f_{2}, N-f_{1},\left(\left\lfloor\frac{N}{f_{1}}\right\rfloor-1\right) f_{2}\right\} \\
& =(n-1) f_{2}
\end{aligned}
$$


and

$$
m<\min \left\{(n-1) f_{2}+n, N,\left\lfloor\frac{N}{f_{1}}\right\rfloor f_{2}\right\}=(n-1) f_{2}+n
$$

and also

$$
\begin{aligned}
& \bar{\Omega}=\min \left\{(n-1) f_{2}+n-1, N-f_{1},\right. \\
& \left.\left(\left\lfloor\frac{N}{f_{1}}\right\rfloor-1\right) f_{2}\right\}=(n-1) f_{2}+n-1 .
\end{aligned}
$$

This implies, if $n_{1}=(n-1) f_{2} \wedge n_{2}=n-1$, that

$$
\begin{gathered}
n_{1}+n_{2}-m+1=\bar{\Omega}-m+1> \\
>(n-1) f_{2}+n-1-\left[(n-1) f_{2}+n\right]+1=0
\end{gathered}
$$

and hence the condition that

$$
\delta_{1}=\max \left\{0, n_{1}+n_{2}-m+1\right\}=0
$$

does not hold for each $n_{1}, n_{2}$ such that $0 \leq n_{1} \leq \bar{m}$ and $0 \leq n_{2} \leq n-1$. Let $n_{1}=(n-1) f_{2} \wedge n_{2}=n-1$; hence

$$
\frac{1}{\left(\begin{array}{c}
m \\
n_{2}
\end{array}\right)} \sum_{0<k \leq \min \left\{n_{1}, n_{2}\right\}}\left(\begin{array}{c}
n_{1} \\
k
\end{array}\right)\left(\begin{array}{c}
m-n_{1} \\
n_{2}-k
\end{array}\right)=\eta
$$

by lemma 2 , where

$$
0 \leq \eta=1-\frac{\left(\begin{array}{c}
n_{1} \\
0
\end{array}\right)\left(\begin{array}{c}
m-n_{1} \\
n_{2}
\end{array}\right)}{\sum_{0 \leq k \leq \min \left\{n_{1}, n_{2}\right\}}\left(\begin{array}{c}
n_{1} \\
k
\end{array}\right)\left(\begin{array}{c}
m-n_{1} \\
n_{2}-k
\end{array}\right)}<1 .
$$

In conclusion, the relationship (6) becomes

$$
\begin{aligned}
& P_{N B} \leq\left(1-\frac{1-\eta}{\bar{m} \cdot(n-1)}\right) . \\
& \sum_{0 \leq n_{1} \leq \bar{m}}\left(\begin{array}{c}
m \\
n_{1}
\end{array}\right)\left(\begin{array}{c}
m \\
n_{2}
\end{array}\right) p^{n_{1}+n_{2}} q^{2 m-n_{1}-n_{2}} \\
& 0 \leq n_{2} \leq n-1
\end{aligned}
$$

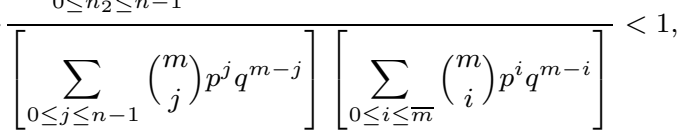

which implies

$$
P_{B}=1-P_{N B}>0 .
$$

Note that the necessary and sufficient condition that we have just proved, given by (8) taken as an equality, is a generalization of the result provided in [4] where the minimum fanout is always set to one.

\section{NUMERICAL RESULTS}

The model and the results derived in the previous sections are now applied to some examples of three-stage networks with size $N=256$.

The first example concerns the scenario in which a nonblocking M-CLOS $\left(N, n, m ; f_{1}, f_{2}\right)$ has $N, n$ and $f_{1}$ fixed (see Fig. 2), while $f_{2}$ can range from a minimum $f_{1}=1$ up to a maximum given by (8). Notice that, given $f_{2}, m$ increases for greater values of $n$. In fact, given all the other constraints,

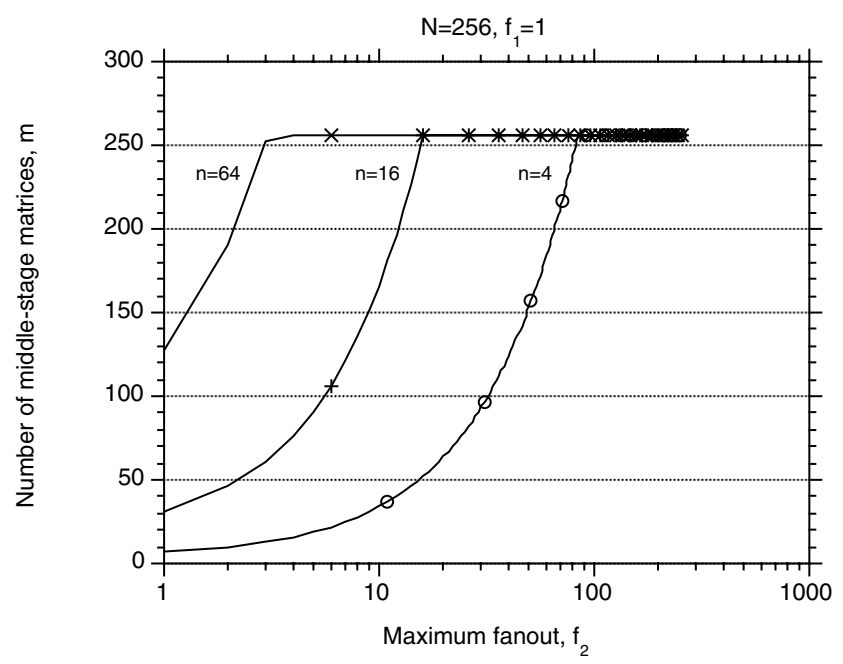

Fig. 2. Configurations of 3-stage nonblocking networks by increasing the maximum fanout

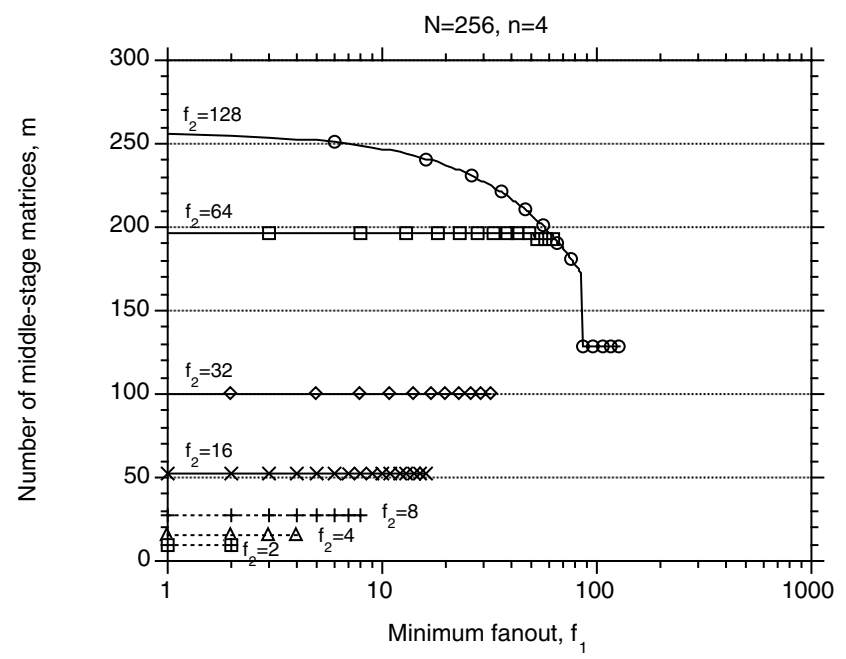

Fig. 3. Configurations of 3-stage nonblocking networks by increasing the minimum fanout

greater values of $n$ imply, on one hand, a greater cardinality of $C_{1, k, i}$, on the other hand, a greater cardinality of $C_{2, h, j}$.

The second application refers to the scenario in which a nonblocking M-CLOS $\left(N, n, m ; f_{1}, f_{2}\right)$ has $N, n$, and $f_{2}$ fixed (see Fig. 3), while $f_{1}$ can range from 1 up to the maximum $f_{2}$. Notice that the number $m$ of second-stage matrices required to satisfy the nonblocking property decreases for greater values of $f_{1}$. Mathematically, it depends on the fact that (8) has several terms decreasing for greater values of $f_{1}$. But the (more interesting) physical reason is that, $f_{2}$ being fixed, the range of a (general) fanout decreases for greater values of $f_{1}$. In other words, increasing $f_{1}$ gives a less general kind of network and, hence, a smaller amount of network resources should be necessary to obtain the nonblocking property.

We compare now the cost of a three-stage network supporting multicast traffic to that of a unicast (point-to-point) network. The crosspoint count of the overall network is 


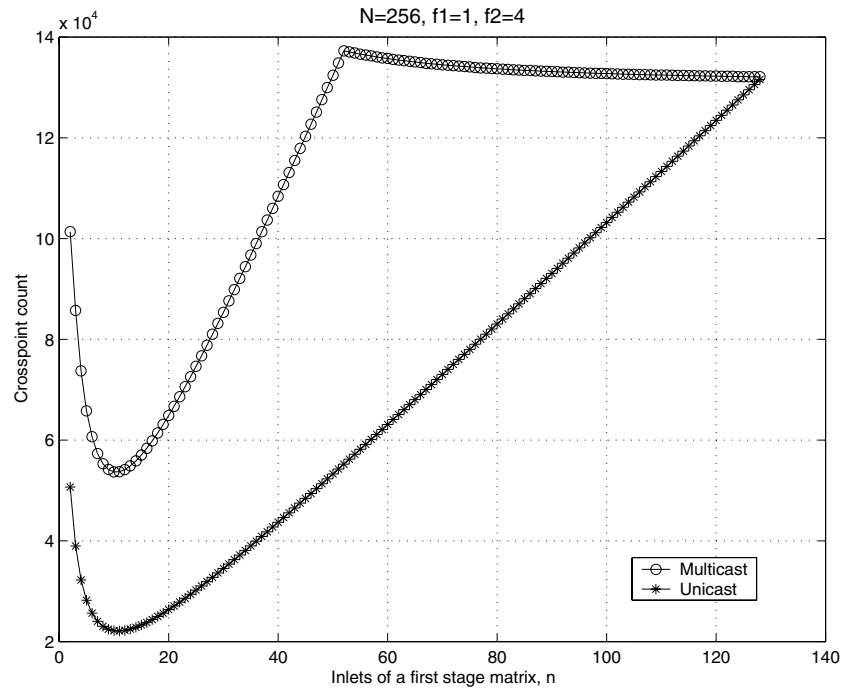

Fig. 4. Number of crosspoints for variable fanout with $f_{2}=4$

assumed to be the cost function ${ }^{1}$.

Fig. 4 considers the case of a fanout ranging from $f_{1}=$ 1 to $f_{2}=4$ and shows that the minimum-cost network is given for the same value $n$ of inlets per first stage matrix. Moreover, unlike the unicast traffic in which the cost grows monotonically beyond the minimum value, the cost function with multicast traffic displays a maximum, after which the cost slightly decreases.

Fig. 5 increases the maximum fanout to $f_{2}=16$. Also in this case the multicast network is more expensive, but, interestingly enough, its minimum cost is attained when the first-stage matrices have maximum size, i.e. $n=128$. Note that for this value the unicast network has maximum cost. Fig. 6 considers the same network assuming now fixed fanout $f_{1}=f_{2}=16$. Differently from the previous cases, now a multicast nonblocking network with maximum size of firststage matrices is cheaper than a unicast network.

Unlike previous models, ours allows us to compute the blocking probability for a multicast three-stage network equipped with a number of second-stage matrices smaller than that required to guarantee nonblocking. This possibility is very useful if the requirement is a quasi-nonblocking network or even a network with a given blocking probability. Apparently the resulting network is cheaper than a nonblocking one. Fig. 7 represents the case of a network with $n=4$ and fanout from $f_{1}=1$ to $f_{2}=4$, with an external offered load ranging from $a=0.2$ to $a=0.8$. Since network nonblocking is provided by $m=16$, the figure shows how the number of secondstage matrices can be reduced, given an offered load and an acceptable blocking probability.

\section{CONCLUSions}

An analytical model has been developed for the analysis of three-stage connecting networks loaded by multicast traffic.

\footnotetext{
${ }^{1}$ A matrix with size $n \times m$ has a cost $n \cdot m$.
}

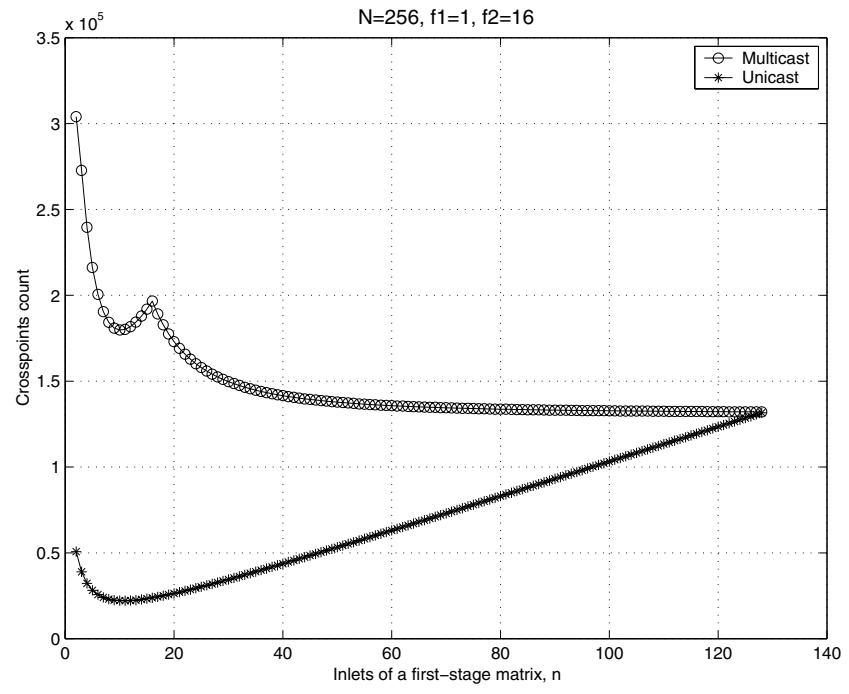

Fig. 5. Number of crosspoints for variable fanout with $f_{2}=16$

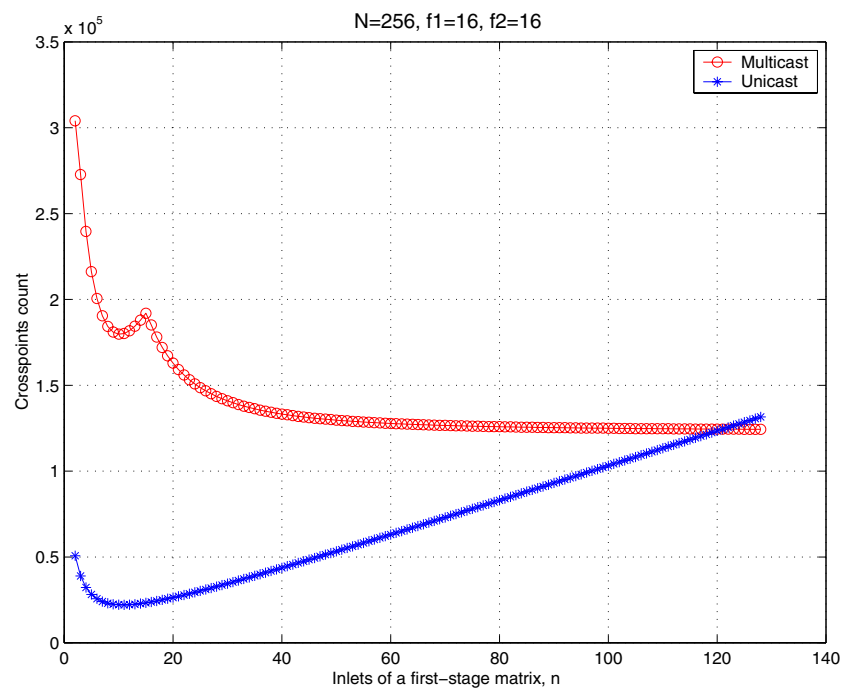

Fig. 6. Number of crosspoints in the network for fixed fanout

Such model has also provided the nonblocking conditions for the network, which have been proven to be both necessary and sufficient. Unlike networks carrying unicast traffic, the cost function of the nonblocking network displays more than one minimum, whose selection to determine the minimum cost network depends on the fanout values. We have shown how the results derived here can be used to dimension a three-stage connecting network with a given blocking probability.

\section{APPENDIX}

Lemma 6: Given $C\left(i_{k, i} ; O_{k, i}\right)$ and $\left|O_{k, i}\right|=f_{k, i}=f_{1}$, then $\delta_{1}=\max \left\{0, n_{1}+n_{2}-m+1\right\}=0$ if

$$
\begin{gathered}
m \geq \min \left\{(n-1) f_{2}+n, N-f_{1}+1,\right. \\
\left.\left(\left\lfloor\frac{N}{f_{1}}\right\rfloor-1\right) f_{2}+1\right\} .
\end{gathered}
$$




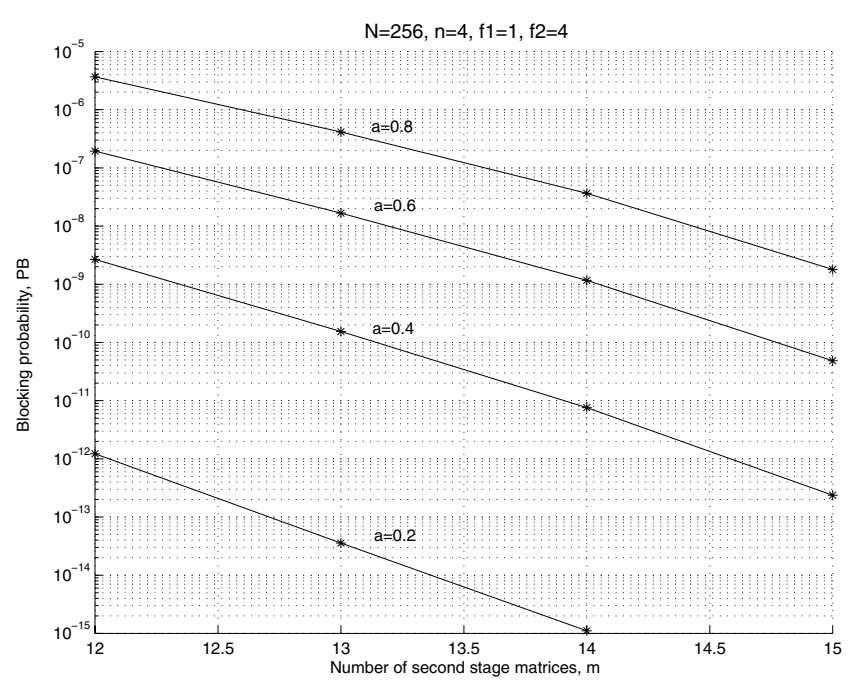

Fig. 7. Blocking probability for different offered load values

Proof: Given $C\left(i_{k, i} ; O_{k, i}\right), f_{k, i}=f_{1}$, by lemma 5

$$
\begin{gathered}
n_{1}+n_{2} \leq \min \left\{(n-1) f_{2}+n-1, N-f_{1},\right. \\
\left.\left(\left\lfloor\frac{N}{f_{1}}\right\rfloor-1\right) f_{2}\right\}=\bar{\Omega} ;
\end{gathered}
$$

then there are three possible cases, i.e.

case 1: $\bar{\Omega}=(n-1) f_{2}+n-1$

case 2: $\bar{\Omega}=N-f_{1}$

case 3: $\bar{\Omega}=\left(\left\lfloor\frac{N}{f_{1}}\right\rfloor-1\right) f_{2}$

that, since

$$
\begin{gathered}
m \geq \min \left\{(n-1) f_{2}+n, N-f_{1}+1,\right. \\
\left.\left(\left\lfloor\frac{N}{f_{1}}\right\rfloor-1\right) f_{2}+1\right\}=\widetilde{m},
\end{gathered}
$$

become nine, i.e.

case 1.1: $\bar{\Omega}=(n-1) f_{2}+n-1 \wedge \widetilde{m}=N-f_{1}+1$

case 1.2: $\bar{\Omega}=(n-1) f_{2}+n-1 \wedge \widetilde{m}=\left(\left|\frac{N}{f_{1}}\right|-1\right) f_{2}+1$

case 1.3: $\bar{\Omega}=(n-1) f_{2}+n-1 \wedge \widetilde{m}=(n-1) f_{2}+n$

case 2.1: $\bar{\Omega}=N-f_{1} \wedge \widetilde{m}=N-f_{1}+1$

case 2.2: $\bar{\Omega}=N-f_{1} \wedge \widetilde{m}=\left(\left\lfloor\frac{N}{f_{1}}\right\rfloor-1\right) f_{2}+1$

case 2.3: $\bar{\Omega}=N-f_{1} \wedge \widetilde{m}=(n-1) f_{2}+n$

case 3.1: $\bar{\Omega}=\left(\left\lfloor\frac{N}{f_{1}}\right\rfloor-1\right) f_{2} \wedge \widetilde{m}=N-f_{1}+1$

case 3.2: $\bar{\Omega}=\left(\left\lfloor\frac{N}{f_{1}}\right\rfloor-1\right) f_{2} \wedge \widetilde{m}=\left(\left\lfloor\frac{N}{f_{1}}\right\rfloor-1\right) f_{2}+1$

case 3.3: $\bar{\Omega}=\left(\left\lfloor\frac{N}{f_{1}}\right\rfloor-1\right) f_{2} \wedge \widetilde{m}=(n-1) f_{2}+n$.

In case 1.1 , since

$$
\begin{aligned}
& \bar{\Omega}=\min \left\{(n-1) f_{2}+n-1, N-f_{1},\right. \\
& \left.\left(\left\lfloor\frac{N}{f_{1}}\right\rfloor-1\right) f_{2}\right\}=(n-1) f_{2}+n-1,
\end{aligned}
$$

it follows that

$$
\bar{\Omega}=(n-1) f_{2}+n-1 \leq N-f_{1} ;
$$

hence

$$
\begin{gathered}
n_{1}+n_{2}-m+1 \leq \bar{\Omega}-\widetilde{m}+1 \leq \\
\leq\left[(n-1) f_{2}+n-1-\left(N-f_{1}\right)\right]+1-1 \leq 0,
\end{gathered}
$$

which proves the lemma in case 1.1.

In case 1.2 , since

$$
\begin{aligned}
& \bar{\Omega}=\min \left\{(n-1) f_{2}+n-1, N-f_{1},\right. \\
& \left.\left(\left\lfloor\frac{N}{f_{1}}\right\rfloor-1\right) f_{2}\right\}=(n-1) f_{2}+n-1,
\end{aligned}
$$

it follows that

$$
\bar{\Omega}=(n-1) f_{2}+n-1 \leq\left(\left\lfloor\frac{N}{f_{1}}\right\rfloor-1\right) f_{2} ;
$$

hence

$$
\begin{gathered}
n_{1}+n_{2}-m+1 \leq \bar{\Omega}-\widetilde{m}+1 \leq \\
\leq\left[(n-1) f_{2}+n-1-\left(\left\lfloor\frac{N}{f_{1}}\right\rfloor-1\right) f_{2}\right]+1-1 \leq 0,
\end{gathered}
$$

which proves the lemma in case 1.2.

In case 1.3., since

$$
\begin{aligned}
& \bar{\Omega}=\min \left\{(n-1) f_{2}+n-1, N-f_{1},\right. \\
& \left.\left(\left\lfloor\frac{N}{f_{1}}\right\rfloor-1\right) f_{2}\right\}=(n-1) f_{2}+n-1,
\end{aligned}
$$

it follows that

$$
\begin{gathered}
n_{1}+n_{2}-m+1 \leq \bar{\Omega}-\widetilde{m}+1 \leq \\
\leq\left[(n-1) f_{2}+n-1-(n-1) f_{2}-n+1\right]+1-1=0,
\end{gathered}
$$

which proves the lemma in case 1.3.

In case 2.1, since

$$
\begin{gathered}
\bar{\Omega}=\min \left\{(n-1) f_{2}+n-1, N-f_{1},\right. \\
\left.\left(\left\lfloor\frac{N}{f_{1}}\right\rfloor-1\right) f_{2}\right\}=N-f_{1},
\end{gathered}
$$

it follows that

$$
\begin{aligned}
& n_{1}+n_{2}-m+1 \leq \bar{\Omega}-\widetilde{m}+1 \leq \\
& \leq\left[N-f_{1}-N+f_{1}\right]+1-1=0,
\end{aligned}
$$

which proves the lemma in case 2.1.

In case 2.2 , since

$$
\begin{gathered}
\bar{\Omega}=\min \left\{(n-1) f_{2}+n-1, N-f_{1},\right. \\
\left.\left(\left\lfloor\frac{N}{f_{1}}\right\rfloor-1\right) f_{2}\right\}=N-f_{1},
\end{gathered}
$$

it follows that

$$
\bar{\Omega}=N-f_{1} \leq\left(\left\lfloor\frac{N}{f_{1}}\right\rfloor-1\right) f_{2}
$$


hence

$$
\begin{gathered}
n_{1}+n_{2}-m+1 \leq \bar{\Omega}-\widetilde{m}+1 \leq \\
\leq\left[N-f_{1}-\left(\left\lfloor\frac{N}{f_{1}}\right\rfloor-1\right) f_{2}\right]+1-1 \leq 0,
\end{gathered}
$$

which proves the lemma in case 2.2.

In case 2.3 , since

$$
\begin{gathered}
\bar{\Omega}=\min \left\{(n-1) f_{2}+n-1, N-f_{1},\right. \\
\left.\left(\left\lfloor\frac{N}{f_{1}}\right\rfloor-1\right) f_{2}\right\}=N-f_{1},
\end{gathered}
$$

it follows that

$$
\bar{\Omega}=N-f_{1} \leq(n-1) f_{2}+n-1 ;
$$

hence

$$
\begin{gathered}
n_{1}+n_{2}-m+1 \leq \bar{\Omega}-\tilde{m}+1 \leq \\
\leq\left[N-f_{1}-(n-1) f_{2}-n+1\right]+1-1 \leq 0,
\end{gathered}
$$

which proves the lemma in case 2.3.

In case 3.1 , since

$$
\begin{gathered}
\bar{\Omega}=\min \left\{(n-1) f_{2}+n-1, N-f_{1},\right. \\
\left.\left(\left\lfloor\frac{N}{f_{1}}\right\rfloor-1\right) f_{2}\right\}=\left(\left\lfloor\frac{N}{f_{1}}\right\rfloor-1\right) f_{2},
\end{gathered}
$$

it follows that

$$
\bar{\Omega}=\left(\left\lfloor\frac{N}{f_{1}}\right\rfloor-1\right) f_{2} \leq N-f_{1}
$$

hence

$$
\begin{gathered}
n_{1}+n_{2}-m+1 \leq \bar{\Omega}-\tilde{m}+1 \leq \\
\leq\left[\left(\left\lfloor\frac{N}{f_{1}}\right\rfloor-1\right) f_{2}-\left(N-f_{1}\right)\right]+1-1 \leq 0
\end{gathered}
$$

which proves the lemma in case 3.1.

In case 3.2 , since

$$
\begin{gathered}
\bar{\Omega}=\min \left\{(n-1) f_{2}+n-1, N-f_{1},\right. \\
\left.\left(\left\lfloor\frac{N}{f_{1}}\right\rfloor-1\right) f_{2}\right\}=\left(\left\lfloor\frac{N}{f_{1}}\right\rfloor-1\right) f_{2},
\end{gathered}
$$

it follows that

$$
\begin{gathered}
n_{1}+n_{2}-m+1 \leq \bar{\Omega}-\tilde{m}+1 \leq \\
\leq\left[\left(\left\lfloor\frac{N}{f_{1}}\right\rfloor-1\right) f_{2}-\left(\left\lfloor\frac{N}{f_{1}}\right\rfloor-1\right) f_{2}\right\rfloor+1-1=0,
\end{gathered}
$$

which proves the lemma in case 3.2.

In case 3.3 , since

$$
\begin{gathered}
\bar{\Omega}=\min \left\{(n-1) f_{2}+n-1, N-f_{1},\right. \\
\left.\left(\left\lfloor\frac{N}{f_{1}}\right\rfloor-1\right) f_{2}\right\}=\left(\left\lfloor\frac{N}{f_{1}}\right\rfloor-1\right) f_{2},
\end{gathered}
$$

it follows that

$$
\bar{\Omega}=\left(\left\lfloor\frac{N}{f_{1}}\right\rfloor-1\right) f_{2} \leq(n-1) f_{2}+n-1 ;
$$

hence

$$
\begin{gathered}
n_{1}+n_{2}-m+1 \leq \bar{\Omega}-\tilde{m}+1 \leq \\
\leq\left[\left(\left\lfloor\frac{N}{f_{1}}\right\rfloor-1\right) f_{2}-(n-1) f_{2}+n-1\right]+1-1 \leq 0,
\end{gathered}
$$

which proves the lemma in case 3.3.

\section{REFERENCES}

[1] C. Clos, "A study of non-blocking switching networks", Bell System Technical Journal, Vol. 32, Mar. 1953, pp. 406-424.

[2] Y. Yang, N. H. Kessler, "Modelling the blocking behaviour of Clos networks", Proc. of DIMACS workshop in Advances in Switching Networks, AMS DIMACS Series, Vol. 42, 1998, pp. 85-102.

[3] C. Y. Lee, "Analysis of switching networks", The Bell System Technical Journal, Vol. 34, No. 6, pp. 1287-1315, Nov. 1955.

[4] F.K. Hwang, S.-C. Liaw, "On nonblocking multicast three-stage Clos networks", IEEE Trans. Networking., Vol. 8, No. 4, Aug. 2000, pp. 535539.

[5] P. Giacomazzi, V. Trecordi, "A Study of Non Blocking Multicast Switching Networks", IEEE Trans. Commun., Vol. COM-43, No. 2/3/4; 1995.

[6] A. Pattavina, G. Tesei, "Multicast non-blocking switching networks", IEEE Trans. Commun., Vol. 50, No. 8, Aug. 2002, pp. 1240-1243.

[7] S. Roman, The Umbral Calculus, Academic Press, 1984. 